\title{
Comment on the determination of Fermi-liquid parameters from cyclotron-wave measurements using empirical procedures
}

\author{
Gordon, R A; Frandsen, J.B.
}

Published in:

Physical Review B

Link to article, DOI:

10.1103/PhysRevB.17.2785

Publication date:

1978

Document Version

Publisher's PDF, also known as Version of record

Link back to DTU Orbit

Citation (APA):

Gordon, R. A., \& Frandsen, J. B. (1978). Comment on the determination of Fermi-liquid parameters from cyclotron-wave measurements using empirical procedures. Physical Review B, 17(6), 2785-2787. https://doi.org/10.1103/PhysRevB.17.2785

\section{General rights}

Copyright and moral rights for the publications made accessible in the public portal are retained by the authors and/or other copyright owners and it is a condition of accessing publications that users recognise and abide by the legal requirements associated with these rights.

- Users may download and print one copy of any publication from the public portal for the purpose of private study or research.

- You may not further distribute the material or use it for any profit-making activity or commercial gain

- You may freely distribute the URL identifying the publication in the public portal 


\title{
Comment on the determination of Fermi-liquid parameters from cyclotron-wave measurements using empirical procedures
}

\author{
R. A. Gordon and J. B. Frandsen* \\ Physics Laboratory I, The Technical University of Denmark, DK-2800 Lyngby, Denmark
}

(Received 29 August 1977)

\begin{abstract}
The application of empirical procedures is shown to lead to a number of ambiguities or errors in the determination of cyclotron-wave dispersion curves. As a result of such ambiguities or errors, it is pointed out that the presently quoted values of the Fermi-liquid parameters $\left\{A_{i}\right\}, i \geq 2$, obtained from cyclotron-wave dispersion curves, can not be considered reliable.
\end{abstract}

It is well known that many-body Fermi-liquid effects have a negligible influence on most of the electrical transport properties of metals. For this reason there has been a strong interest in cyclotron-wave measurements since it has been suggested that the propagation of such waves might be appreciably affected by the Fermi-liquid interaction in the long-wavelength limit. ${ }^{1-7}$ It has been shown, however, that the empirical analysis of cyclotron-wave data which has generally been used to estimate the Fermi-liquid parameters is necessarily incorrect since the effects of a finite relaxation time on the cyclotron-wave propagation have not been taken into account properly. ${ }^{8-10}$ More recently, it has been suggested by other authors, on the basis of a different empirical analysis, that the propagation of cyclotron waves can be accounted for entirely by finite-relaxation-time effects without including the Fermi-liquid interaction. ${ }^{11}$ In view of the theoretical significance attached to the observability of Fermi-liquid effects in metals and the fact that different empirical methods can lead to substantially different conclusions regarding the existence of such effects, it is of considerable importance to examine more carefully the assumptions made in the empirical methods themselves. It is the purpose of this Comment to present such an examination. In particular, it is shown that both methods possess serious ambiguities and inconsistencies and that no reliable conclusion regarding the existence of Fermi-liquid effects in metals can be given using such methods.

The empirical determinations of the cyclotronwave dispersion curves which have been proposed are based on the fact that cyclotron waves propagating across a finite-thickness sample will interfere constructively or destructively with a second background signal at values of the magnetic field corresponding to the inclusion or exclusion of an additional half cyclotron wavelength in the sample, i.e., experimental extrema will be observed at magnetic fields for which the phase resonance con- dition

$$
\operatorname{Re}(q d)=\pi(n+1) ; n=0,1,2, \ldots
$$

is satisfied. Here, $q$ is the cyclotron-wave vector which is magnetic-field dependent and $d$ is the sample thickness. The use of Eq. (1) to obtain a cyclotron-wave dispersion curve assumes that any ambiguities or magnetic-field variations in the phase of the background signal can be eliminated, e.g., by requiring that measurements taken at different frequencies fall on a common dispersion curve. This is the approach taken by Walsh et al. in the bulk of the published work dealing with the determination of Fermi-liquid effects from cyclotron-wave data. ${ }^{3-7}$ No description has ever been published as to how the experimental points should be made to lie on the common dispersion curve or what form the common dispersion should take. However, the fact that it has been clearly shown that no common dispersion exists for the finite relaxation times present in the cyclotron-wave experiments casts serious doubts as to the reliability of such an approach. ${ }^{8-10}$ Even more serious, as noted above, is that such an empirical approach is internally inconsistent and necessarily leads to incorrect results. Thus, for example, the published value of the Fermi-liquid parameter $A_{3}=0$ obtained from such an empirical analysis necessarily implies that the empirically obtained dispersion curve should agree with the theoretical free-electron dispersion curve in the long-wavelength limit for extraordinary cyclotron waves propagating above the cyclotron resonance fundamental field_-in striking contrast to what is actually observed as can be seen from Fig. 1. This long-wavelength discrepancy is particularly serious since it is precisely the long-wavelength portion of the cyclotron-wave dispersion curve which is used by Walsh et al. to determine the Fermi-liquid parameters. Equally large unexplained discrepancies also exist for the other higher-order Fermi-liquid parameters $\left\{A_{i}\right\}$, $i=3,4,5$, and it must therefore be concluded that 


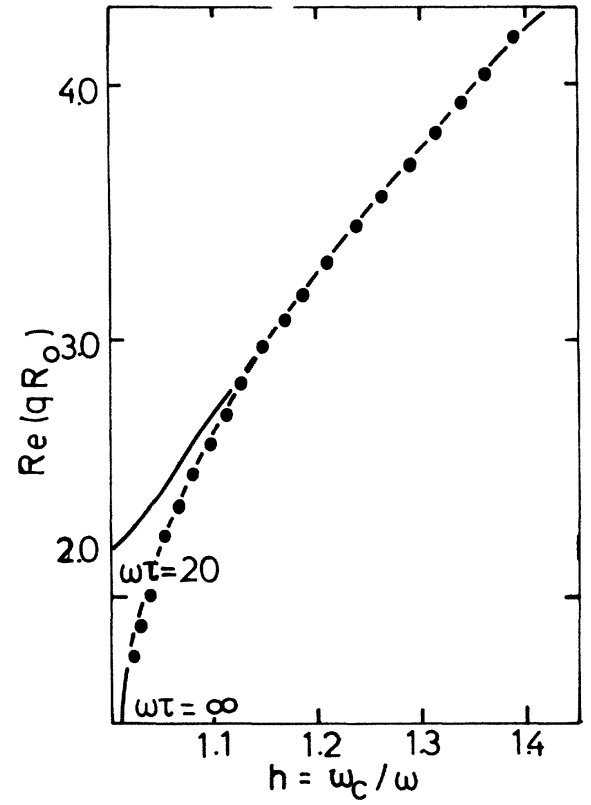

FIG. 1. Theoretical free-electron dispersion curves for extraordinary cyclotron waves at $17.35 \mathrm{GHz}$ above the cyclotron resonance magnetic field in potassium. The black circles denote the empirically determined points of Walsh et al. at $17.4 \mathrm{GHz}$ (see Ref. 3), while $\tau$ and $\omega_{c}$, respectively, are the relaxation time between electron scattering events and the cyclotron frequency.

either the published values of $\left\{A_{i}\right\}=0, i \geqslant 3$, or the empirically determined dispersion curve used to find them, or both, are incorrect, i.e., not even the convergence of a Fermi-liquid expansion can be decided by such an empirical method. Another important deficiency of the empirical method of Walsh et al. is the extremely large ambiguity which is inherent in the empirical method itself even in cases where the effects of a finite relaxation time are negligble or simply ignored. This is well illustrated by the fact that Walsh et al. have used their empirical method to fit the same set of experimental data not only to the dispersion curve on which their estimate of the Fermi-liquid parameter $A_{2}$ is based, but also to a second dispersion curve differing by a factor of two or more in cyclotronwave vector as well. ${ }^{12-14}$ That ambiguities of such a large order of magnitude can so readily occur in the empirical determination of a cyclotron-wave dispersion curve raises serious questions as to the efficacy of such a method in determining Fermi-liquid parameters which would be expected $a$ priori to lead to changes of a few percent at most in the free-electron wave vector. Thus, no unambiguous information about any of the Fermi-liquid parameters accessible to cyclotron-wave measurements $\left\{A_{i}\right\}, i \geqslant 2$ can be obtained by the empirical method of Walsh et al.

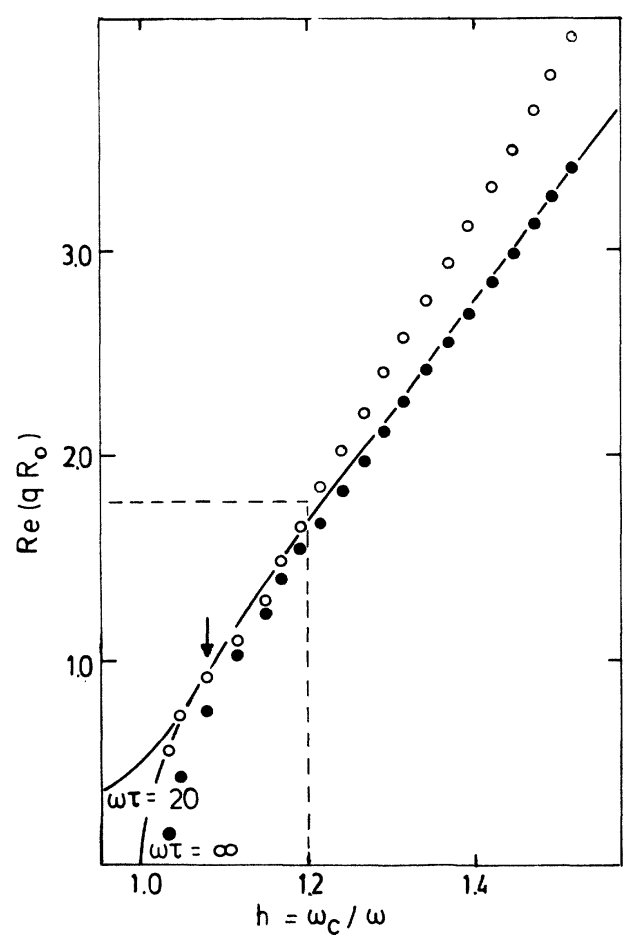

FIG. 2. Empirically determined cyclotron-wave dispersion curves in potassium using the method of Walsh et al. (black circles, see Ref. 3) and the method of Særmark and Lebech (open circles, see Ref. 11 and text). The dashed lines indicate the limited range of magnetic fields shown by the latter authors (Fig. 7 in Ref. 11) while the smooth curves denote theoretical cyclotron-wave dispersion curves calulated using a free-electron dispersion curve. Here, $\tau$ and $\omega_{c}$, respectively, are the relaxation time between electron scattering events and the cyclotron frequency.

Faced with this discrepancy, Særmark and Lebech have recently proposed a different empirical method of analyzing the cyclotron-wave data in which an arbitrary experimental point is taken to lie along the free-electron dispersion curve. The remaining experimental points are then assumed to be separated by the same distance

$$
\Delta \operatorname{Re}\left(q R_{0}\right)=\left(R_{0} / d\right) \Delta \operatorname{Re}(q d)=\pi v_{F} / \omega d
$$

along the $q R_{0}$ coordinate axis where $v_{F}$ is the Fermi velocity, $\omega$ is the angular frequency, and $R_{0}$ $\equiv v_{F} / \omega .{ }^{11}$ It is claimed that such a procedure will lead to agreement with the free-electron dispersion curve. This is not the case, however, if the procedure is continued beyond a limited range of magnetic fields centered about the arbitrary point itself indicated by the arrow in Fig. 2. ${ }^{15}$ Equally important is the fact that this particular discrepancy is not fixed but varies greatly depending on which of the arbitrary points is taken to lie along the free-elec- 
tron dispersion curve. Another difficulty is the large ambiguity introduced by the use of an arbitrary point to determine the cyclotron-wave dispersion curve. For example, the use of such an arbitrary point has, on other occasions, led to the fitting of cyclotron-wave data to a nonexistent branch of the dispersion curve. ${ }^{16}$

In short, the ambiguities and uncertainties in the empirical method which has generally been used to analyze cyclotron-wave data, as well as another method recently suggested, are sufficiently serious that it must be concluded that no reliable estimate of the Fermi-liquid parameters based on such methods can be made. In addition, the magneticfield variation of the background signal in the vicinity of the cyclotron resonance fundamental and its higher harmonics is sufficiently large that the only unambiguous method of obtaining a cyclotronwave dispersion curve suitable for the determination of Fermi-liquid parameters will be by a direct comparison of the experimental data with the theoretical solution of the boundary-value problem for the excitation of cyclotron waves in the actual finite-sample geometry. Such a boundary-value problem must take into account both finite relaxation time and Fermi-liquid effects using different boundary conditions since recent work has shown that both the cyclotron resonance background and the cyclotron-wave excitation are sensitive to the nature of electron scattering at the surface of a metal. ${ }^{17,18}$ A calculation of this type would entail a good deal of computational difficulty but until such a calculation can be made, the values of the Fermi-liquid parameters which abound in the literature can not be considered to be even qualitatively correct.

Note added in proof. An approximate solution to the boundary-value problem including both Fermiliquid and finite- $\omega \tau$ effects has recently been obtained by Fredkin and Dunifer for the transmission of ordinary cyclotron waves through a finite potassium sample. The values of the Fermi-liquid parameters $A_{2}$ and $A_{3}$ obtained in this way (i.e., $\left.\left|A_{2}\right|=0.03,\left|A_{3}\right|=0.014\right)$ differ significantly from the value of $\left|A_{2}\right|=0.022 \pm 0.002$ quoted by Walsh et al. (Ref. 6) and are completely at variance with the zero values for $A_{3}$ and $A_{2}$ quoted by Walsh et $a l$., and Særmark and Lebech, respectively.
*Present address: Computer Research Division, Regnecentralen, Hovedvejen 9, DK-2600 Glostrup, Denmark.

${ }^{1}$ N. D. Mermin and Y. C. Cheng, Phys. Rev. Lett. 20, 839 (1968).

${ }^{2}$ S. C. Ying and J. J. Quinn, Phys. Rev. 180,193 (1969).

${ }^{3}$ P. M. Platzman, W. M. Walsh, Jr., and E-Ni Foo, Phys. Rev. 172, 639 (1968).

${ }^{4}$ W. M. Walsh, Jr., and P. M. Platzman, Phys. Rev. Lett. 15, 784 (1965).

${ }^{5}$ P. M. Platzman and W. M. Walsh, Jr., Phys. Rev. Lett. 19, 514 (1967).

${ }^{6}$ W. M. Walsh, Jr., L. W. Rupp, Jr., P. H. Schmidt, and R. N. Castellano, Bull. Am. Phys. Soc. 18, 336 (1973).

${ }^{7}$ P. M. Platzman and P. A. Wolff, in Waves and Interactions in Solid State Plasmas, Supplement No. 13 (Academic, London, 1973), Chaps. 8 and 10.

${ }^{8} \mathrm{~J}$. B. Frandsen and R. A. Gordon, Phys. Rev. B 14, 4342 (1976).

${ }^{9}$ D. R. Fredkin and A. R. Wilson, in Proceedings of the Eleventh International Conference on Low Temperature Physics, edited by J. F. Allen, J. F. Finlayson, and D. M. McCall (University of St. Andrews, St. Andrews, Scotland, 1968), Vol. 2, p. 1178.

${ }^{10} \mathrm{~J}$. B. Frandsen, in Proceedings of the Fourteenth International Conference on Low Temperature Physics, edited by M. Krusius and M. Vuorio (North-Holland, Amsterdam, 1975), Vol. 4, p. 321.

${ }^{11}$ K. Særmark and J. Lebech, Phys. Status Solidi B 79, 723 (1977).

${ }^{12}$ P. M. Platzman and W. M. Walsh, Jr., Phys. Rev. Lett. 20, 89 (1967).

${ }^{13}$ W. M. Walsh, Jr. in Solid State Physics: Electrons in Metals, edited by J. F. Cochran and R. R. Haering (Gordon and Breach, London, 1968), Vol. 1, p. 225ff.

${ }^{14}$ D. C. Hamilton and A. L. McWhorter, Bull. Am. Phys. Soc. 13, 438 (1968).

${ }^{15}$ The deviation in Fig. 2 is accentuated by the fact that Særmark and Lebech have used the wrong value $\left(v_{F} / c=3 \times 10^{-3}\right.$ in Ref. 11) for the Fermi velocity of potassium and, hence, a wrong scaling factor, viz., Eq. (2). The use of the correct Fermi velocity $\left(v_{F}=0.71 \times 10^{6} \mathrm{~m} / \mathrm{sec}\right)$ is not, however, sufficient to give agreement with the free-electron dispersion curve. Such deviations are especially serious in the "linear" portion of the cyclotron-wave dispersion curves where both Fermi-liquid and finite- $\omega \tau$ effects are negligible.

${ }^{16}$ Such a procedure has been adopted in choosing an experimental extremum in transmission measurements in silver to lie along the second branch of the extraordinary cyclotron-wave dispersion curve [K. Særmark and J. Lebech, Phys. Status Solidi B 72, 375 (1975), Fig. 4al. In a range of magnetic fields for which the damping of the cyclotron waves across the sample was so large (at least $450 \mathrm{~dB}$ ) as to have precluded the possibility that the transmitted signal actually measured could have been due to cyclotron waves at all; J. B. Frandsen, thesis. The Technical University of Denmark, Physics Laboratory I, Report No. 149 , (unpublished).

${ }^{17}$ D. E. Zherebchevskii and E. A, Kaner, Zh. Eksp. Teor. Fiz. 63, 1858 (1972)[Sov. Phys.-JETP 36, 983 (1973)].

${ }^{18}$ R. A. Gordon and J. B. Frandsen, Solid State Commun. 24, 221 (1977). 